\title{
Adjuvant Therapy of Triple Negative Breast Cancer
}

\author{
Edith A. Perez, M. D., Alvaro Moreno-Aspitia, M.D., E. Aubrey Thompson, Ph. D., and Cathy \\ A. Andorfer, Ph.D. \\ Mayo Clinic, 4500 San Pablo Road, Jacksonville, FL 32224, USA
}

\begin{abstract}
Patients with the triple negative subtype of breast cancer have an overall poor outcome, with earlier relapses, distinct patterns of metastases, and lack of specific targets for treatment selection. Classification of these tumors has begun to be modified by inclusion of immunohistochemistry for various markers, and gene profiling, to further characterize this subtype of breast cancer, may aid in the identification of new targeted therapies. Anthracyclines and taxanes remain the standard of care in the adjuvant setting. However, novel anti-angiogenesis, anti-tubulin, and DNA repair agents are already under evaluation in (neo) adjuvant trials. Molecular characterization is being included in trials to identify optimal adjuvant strategies. The aim of this manuscript is to review data concerning the molecular characterization of triple negative breast cancers as well as the clinical outcomes of treating patients with existing adjuvant treatments, and to highlight newer adjuvant research strategies in development.
\end{abstract}

\section{Keywords}

Breast cancer; triple negative; basal-like breast cancer; adjuvant therapy

\section{Introduction}

Triple negative breast cancers represent a heterogeneous group of diseases, characterized by significant variability in morphological and pathological features. These tumors lack the three most significant therapeutic markers for clinical management of breast cancer patients: human epidermal growth factor receptor 2 (HER2), estrogen receptor-alpha (ER), and progesterone receptor (PR), and are thus labeled as "triple negative" (TN). They account for at least $15-20 \%$ of all breast cancers. Epidemiologic studies illustrate a higher prevalence of TN tumors among younger women and those of African descent [1-3]. Clinicopathologic features of TN breast cancers include younger age at onset, larger mean tumor size, and higher grade and incidence of node positivity at presentation compared to what is expected based on tumor size (Table 1). Additionally, patients with TN tumors have a high probability of early tumor relapse after diagnosis, increased propensity to develop brain metastases, and rapid risk of death after tumor relapse (even after appropriate locoregional management), thus identification of the best adjuvant therapies is a focus of intense research [4-10].

The majority of TN breast carcinomas are ductal in origin; however, several other aggressive phenotypes appear to be overrepresented, including metaplastic, atypical or typical medullary, and adenoid cystic [11]. Reports related to incidence of brain metastases

Corresponding author: Edith A. Perez, M. D., Serene M. and Frances C. Durling Professor of Medicine, Mayo Clinic, 4500 San Pablo Road, Jacksonville, FL 32224 USA, Tel: 1-904-953-0118, Fax: 1-904-953-1412, perez.edith@ mayo.edu. 
and outcome from three institutions [6-8] are particularly noteworthy in defining the higher incidence and worse outcome after brain metastases in patients with TN disease. These malignancies represent a major challenge for physicians and patients, both in the context of understanding its molecular basis and optimization of patient management.

Interpretation of data from various studies reporting the natural history and response to existing therapies is partially confounded by the fact that there is a lack of universally accepted definitions for estrogen or progesterone positivity. Some reports describe it as immunohistochemistry positivity in $>1 \%$ of stained cells, others define it as $>10 \%$ staining, still others use a combination of percent and strength of staining. Moreover, other investigators advocate mRNA or gene expression analysis to define positivity. The most commonly used definition in the studies reviewed and ongoing adjuvant trials use protein expression in $>10 \%$ of cells to define ER and PR positivity. Tumor characterization is further confounded by the challenge of HER2 testing, in terms of whether to test for protein expression or gene amplification, definitions of positivity, and accuracy of testing [12-14].

The advent of molecular profiling has significantly altered the diagnostic and therapeutic landscape for TN breast cancer, which we discuss in this manuscript.

\section{Methods}

PubMed manuscripts and meeting abstracts from 2005 to September 2009 under the headings of "triple negative breast cancer", "basal cell breast cancer," and "human basal breast cancer" as well as the http://clinicaltrials.gov database were reviewed.

\section{Results}

\section{Molecular Characterization}

There is strong evidence that at least five broad categories of breast cancer can be identified based on intrinsic gene expression patterns $[15,16]$. These categories include luminal A and luminal B tumors (which are primarily ER-positive) HER2-enriched tumors, basal-like tumors, and the so-called normal-like tumors. There is a tendency to equate basal-like tumors with TN breast cancer [5, 17-21], since basal-like tumors are ER/PR-negative and do not exhibit HER2 amplification and/or overexpression. A more detailed evaluation reveals that most TN tumors express basal/myoepithelial cell specific cytokeratins (CK5/6, CK14, and CK17), vimentin, epidermal growth factor receptor (EGFR), and markers of a high proliferative state $[15,16,22-24]$. These features constitute the molecular hallmarks of the basal-like tumor intrinsic subgroup. However, as many as one-third of tumors identified as TN exhibit a non-basal genomic profile [25], and it has been reported that about 1700 genes are differentially expressed when CK5/CK14-positive ER-negative tumors were compared to CK5/CK14-negative ER-negative tumors [26]. TN tumors that do not manifest the typical basal-like molecular signature generally have a better prognosis, particularly among those tumors that express activation of complement, immune responsiveness, androgen receptor, and ER responsive genes such as GATA3, TFF1, and DNALI1 [27]. Such observations indicate that TN breast cancers exhibit a range of molecular and clinical properties, and furthermore that $\mathrm{TN}$ tumors are actually not a single type, but rather comprised of several subtypes with different molecular characteristics, natural histories, and responsiveness to treatment (Figure 1) [9, 28-31].

Older classification methods are being supplanted by molecular characterization utilizing immunohistochemistry or gene profiles $[17,18,21]$. The rationale for this evolution is based on better defining the heterogeneity within the TN breast cancer cohort and on providing prognostic and predictive information to inform treatment decisions. The majority of 
therapeutic targets currently under investigation fall within three broad categories: antiangiogenesis, stabilization of microtubules and deoxyribonucleic acid (DNA) repair [32, 33] (Figure 2).

Several molecules integrally involved in DNA repair are aberrantly expressed in TN breast cancer, which may have implications for chemotherapy sensitivity [9, 25-27, 29-31]. Comparative genomic hybridization studies indicate that basal-like tumors have characteristic increased DNA copy number alterations, consistent with genomic instability [34]. Several additional and targetable molecular pathways implicated in the pathogenesis of basal-like breast cancer include the mitogen-activated protein kinase (MAPK) pathway, the AKT pathway, p53 mutations (reported in $40-80 \%$ of cases), and the poly adenosine diphosphate ribose polymerase 1 (PARP1) pathway. The extent to which the breast cancer gene 1 (BRCA1) pathway contributes to the behavior of sporadic basal-like breast cancers is an area of active research.

\section{Outcome to Standard Therapies}

Although neither endocrine nor anti-HER2 therapies are viable treatment options for patients with TN disease, several studies document that these tumors are sensitive to neoadjuvant therapies - mostly including anthracyclines and taxanes [24, 28]. In several studies, these tumors have demonstrated greater response to neoadjuvant chemotherapy compared to other breast tumor types. However, somewhat paradoxically, in spite of initial responsiveness, these patients have an unacceptable risk of tumor relapse and mortality $[18,35]$.

For example, patients with $\mathrm{TN}$ breast cancer had a higher pathological response to neoadjuvant anthracycline-based treatment administered at Peking University People's Hospital (38\%) compared to $12 \%$ in those with non-TN disease, $\mathrm{p}=0.002$, but overall decreased disease-free survival rates $(\mathrm{p}=0.004)$ [24]. These investigators did note that if pathologic complete response (pCR) was achieved, patients with TN breast cancer and non$\mathrm{TN}$ breast cancer had similar survival ( $\mathrm{p}=0.497$ ). However, patients who did not exhibit $\mathrm{pCR}$ had significantly worse survival if they had TN tumors compared with non-TN breast cancer $(p<0.05)$ [24]. Similar data of poor overall outcome to anthracycline-based adjuvant treatment was reported by Tan, et al $[19,36]$. Liedtke, et al. described outcome to anthracycline plus taxane-based neoadjuvant treatment for patients treated at M. D. Anderson Cancer Center, consistent with a higher pathological complete response in TN vs. non-TN breast cancer ( 22 vs. $11 \%$ respectively, $\mathrm{p}=0.034$ ), but decreased three-year progression-free survival rates $(\mathrm{p}<0.0001)$ and overall survival $(\mathrm{OS})$ rates $(\mathrm{p}=0.027)$ [37]. Of interest was that recurrence and death rates were higher for TN breast cancer but only in the first 3 years; if $\mathrm{pCR}$ was achieved, both cohort of patients had similar survival ( $\mathrm{p}=0.027)$; but those with residual disease at surgery had a worse outcome compared to those with nonTN breast cancer $(\mathrm{p}=<0.0001)$ [24].

Pre-clinical data suggest that TN tumors may have increased sensitivity to agents whose mechanism of action involve DNA repair. Platinum compounds act by intercalating into DNA and non DNA targets and causing induction of cell death via the inhibition of transcription and/or DNA replication mechanisms [38]. A few studies evaluating platinum agents in advanced patients with TN breast cancer in the metastatic setting have been performed. However, it remains unclear whether such drugs particularly target this subtype of breast cancer. A retrospective study from Korea looking at the outcomes of patients with TN metastatic breast cancer in comparison with patients with other subtypes, all treated with a taxane plus platinum containing regimens, demonstrated no difference in the response rate among these 2 groups of patients (37.5 vs. 38.5\%) [39]. However, patients with TN breast cancer had a shorter time to death after chemotherapy (19 vs. 50 months, $p=0.037)$ and overall survival ( 21 vs. 56 months, $\mathrm{p}=0.030$ ). A similar retrospective study from the United 
Kingdom [40] demonstrated that patients with advanced TN breast cancer treated with platinum-based chemotherapy had overall response rates that were similar to those with nonTN tumors ( $41 \%$ vs. $31 \% ; \mathrm{p}=0.3$ ), but, in this study, these patients had a longer progression free-survival ( 6 vs. 4 months; $p=0.05$ ) and similar OS (11 vs. 7 months; $p=0.1)$ than the other patients. Additional prospective trials are needed to determine the true role of platinum compounds in patients with TN tumors. One such trial is the phase III randomized Triple Negative Trial (TNT), conducted but the European National Cancer Research Institute (NCRI), in patients with metastatic TN disease ( $\mathrm{N}=370-450)$, who will be randomized to either 6 cycles of carboplatin or 6 cycles of docetaxel as first-line therapy with a cross over design upon progression (NCT00532727) [41].

Two neoadjuvant studies suggest that platinum-based regimens have a potentially important role to play in chemotherapy naive patients with TN breast cancer. A pre-operative NCCTG study (N0338) of dose dense (dd) docetaxel and carboplatin $\times 4$ cycles in 57 patients with locally advanced breast cancer demonstrated a clinical response of $75 \%$ (15 cCR, $28 \mathrm{cPR}$ ), including a pCR rate of $16 \%(9 / 57)$. However, the pCR for patients with TN tumors was $43 \%$ (4/9) [42]. The other study provided 4 cycles of single agent cisplatin in 28 patients with stage II or III TN tumors and reported a pCR of $22 \%$ (6/28). Additional neoadjuvant clinical trials with platinum compounds in combination with other drugs targeting patients with TN disease are currently ongoing. These include the phase II studies of cisplatin with the novel PARP-1 inhibitor AZD-2281 (NCT00782574) [43]; gemcitabine and carboplatin in combination with the PARP-1 inhibitor BSI-201 (NCT00813956) [44]; paclitaxel plus carboplatin in combination with the multikinase inhibitor sutinib (NCT00887575) [45]; and docetaxel plus carboplatin in combination with the HER1/EGFR inhibitor erlotinib (NCT00491816) [46].

A particular group of patients with $\mathrm{TN}$ tumors that may respond better to the platinums are those patients with BRCA1 mutation. BRCA1 is a tumor suppressor gene which, when mutated, is associated with the development of hereditary breast cancers. Most breast cancers that develop in patients with a BRCA1 mutation are of the TN phenotype. However, loss of BRCA1 can also be observed in sporadic tumors [47, 48]. Sporadic basal-like breast cancer tumors are characterized by the dysfunction of the BRCA1 pathway caused by BRCA1 gene promoter methylation, BRCA1 transcriptional inactivation, or both [48-51]. BRCA1 expression is important in DNA repair, transcriptional regulation, and activation of cell-cycle checkpoints, ubiquitination and maintenance of chromosomal stability [52]. Preclinical studies indicate that tumors with BRCA1 dysfunction harboring deficient doublestranded DNA break repair mechanisms are sensitive to agents that cause DNA damage, such as platinum agents.

The observation that TN tumors overexpress the epidermal growth factor receptor (EGFR) led to the development of two phase II studies investigating the activity of cetuximab, a monoclonal anti-EGFR antibody, in patients with metastatic TN breast cancer. The first phase II study targeted 102 patients with previously treated disease with the combination of cetuximab and carboplatin. This regimen achieved an overall response rate of $18 \%$ and an overall clinical benefit rate of $27 \%$. Unfortunately, time to progression was short ( 2 months), and OS was only 12 months [53]. The other study reported the preliminary results of a randomized phase II trial that investigates the combination of irinotecan and carboplatin with or without cetuximab in 103 patients also with advanced TN disease [54]. This study reported that the combination of chemotherapy plus cetuximab led to a higher response rate ( $49 \%$ vs. $30 \%$ ) but it was associated with a much greater incidence of grade 3-4 adverse events. Currently there is a neoadjuvant phase II study in development evaluating the combination of docetaxel and cetuximab in patients with TN breast cancer (the TENEO study; NCT00600249) [55]. 
An agent that may have particular activity in patients with TN tumors is an MSA called ixabepilone. This is the first epothilone B-analog approved for the treatment of patients with metastatic breast cancer. A pooled analysis of data from 399 patients with TN tumors who participated in two phase III trials of ixabepilone plus capecitabine demonstrated this regimen achieved an ORR of $31 \%$ and a median PFS time of 4.2 months [56]. These results were similar to those obtained in patients with non-TN metastatic breast cancer and support the use of this agent in the adjuvant PACS-08 trial.

\section{Newer Approaches}

Novel adjuvant therapy approaches are guided by data in preclinical models, molecular profiling, and results of trials in metastatic disease. A variety of well planned adjuvant clinical trials concentrating on patients with $\mathrm{TN}$ disease are ongoing, investigating the role of the MSA agent ixabepilone, platinum agents, and anti-VEGF approaches.

The PACS 08 [57] adjuvant trial builds on the data from the PACS 01 and preclinical/ neoadjuvant/metastatic data with ixabepilone $[56,58,59]$. This is a multi-cooperative group randomized phase III study of patients with TN (or ER- HER- PR+ if tumors $>2 \mathrm{~cm}$ ), randomized to three cycles of FEC ( 5 - fluorouracil + epirubicin + cyclophosphamide) followed by either three doses of docetaxel or ixabepilone, each dose every three weeks.

The phase III adjuvant TITAN [60] trial evaluates four cycles of standard doxorubicin plus cyclophosphamide followed by either four cycles of ixabepilone or 12 doses of weekly paclitaxel [61].

The GEICAM 2006-03 [62] is a randomized phase II neoadjuvant study and includes two sub studies [63]. First, a sample of the primary tumor is analyzed by immunohistochemistry (for cytokeratins and ER/PR/HER2). Second, and depending on the expression of these markers, the patients are characterized as having either luminal A or basal subtype of breast cancer, with random assignment to a standard or experimental treatment. The randomization for those assigned to the basal phenotype is four doses standard epirubicin + cyclophosphamide every 3 weeks followed by either four doses of docetaxel or docetaxel plus carboplatin.

The North American Intergroup study CALGB 40603 [64] is a randomized phase II neoadjuvant trial of 12 doses of paclitaxel at $80 \mathrm{mg} / \mathrm{m}^{2}+/$ - four doses of carboplatin at AUC $=6+/-$ nine doses of bevacizumab $10 \mathrm{mg} / \mathrm{kg}$, followed by four doses of dd doxorubicin + cyclophospamide [65].

The phase III adjuvant BEATRICE study [66] uses a fairly pragmatic approach to evaluate various chemotherapy agents with or without bevacizumab at a $5 \mathrm{mg} / \mathrm{kg}$ per week "equivalent" (in other words $10 \mathrm{mg} / \mathrm{kg}$ when used every two weeks, or $15 \mathrm{mg} / \mathrm{kg}$ when used every three weeks) for patients with TN breast cancer.

Accrual to these studies is critical to determining whether any of these agents should supplant or be routinely added to anthracyclines and taxanes. Added to the already active adjuvant portfolio are two facts: 1. these trials are in general accompanied by collection of tumor and blood specimens (important for translational research) and 2. there are several neoadjuvant and metastatic trials targeting novel approaches (such as PARP and histone deacetylase (HDAC inhibitors) which we hope will demonstrate enough anti-tumor activity to warrant subsequent adjuvant evaluation.

Of the areas of significant novel interest, both the Notch-survivin gene pathway and PARP arguably deserve higher consideration. Immunohistochemistry and genetic signature 
evaluations of TN breast cancer have identified that activated Notch-1 is preferentially expressed in breast cancer, segregates with basal-like disease and is correlated with decreased survival $[27,67]$. It has been proposed that upregulation of survivin via a Notch dependent mechanism may suppress apoptosis, disrupt cell cycling and possibly promote resistance to common therapeutics such as taxanes and platinum compound [27].

Additionally since Notch is proposed to have a role in the differentiation and maintenance of mammary progenitor/stem cells [68], a Notch-survivin pathway may contribute to higher recurrence rates. Thus, Notch and survivin antagonists are logical agents to be evaluated.

PARP is a nuclear enzyme that signals or detects the presence of DNA damage by catalyzing the addition of ADP-ribose units to DNA, histone and various DNA repair enzymes, and also by facilitating DNA repair $[13,69,70]$. In vitro and in vivo models have demonstrated that PARP inhibitors potentiate the activity of DNA-damaging agents such as alkylators, platinums and topoisomerase inhibitors. Tumors with DNA repair defects (such as those with BRCA mutations or basal-like tumors with dysfunctional BRCA activity) may be more sensitive to PARP inhibition. A recently presented randomized phase II trial of patients with advanced TN tumors treated with carboplatin and gemcitabine alone or in combination with the PARP-1 inhibitor BSI-201, demonstrated that this novel agent significantly improved ORR ( $48 \%$ vs. $16 \%, \mathrm{p}=0.002)$, PFS (6.9 months vs. 3.3 months, HR $=0.342, \mathrm{p}<0.0001)$, and median OS (9.2 months vs. 5.7 months, $\mathrm{HR}=0.348, \mathrm{p}=0.0005)$ when added to the chemotherapy. This data has now led to an upcoming phase III trial. Studies with other PARP-1 inhibitors such as AGO14699, AZD-2281 and ABT-888 are ongoing or in development. It is important to note that defects in homologous recombination repair can also be caused by loss of function of proteins other than BRCA1 and BRCA2, potentially widening the utility of this therapeutic strategy. Additionally, epigenetic events can cause some sporadic tumors to appear as phenocopies of BRCA1 or BRCA2 deficient tumors without actually possessing germline mutations in either of these genes (Pres JZ, 2008), again increasing the potential efficacy of this line of therapy.

\section{Conclusion}

These so-called TN tumors (HER2-/ER-/PR-) are currently best treated with conventional adjuvant or neoadjuvant anthracycline and taxane chemotherapeutic drugs. Although significant initial responses are often observed, disease-free survival is significantly reduced in this type of cancer patients. Ongoing neoadjuvant and adjuvant trials are evaluating novel anti-tubulin agents such as ixabepilone, the platinums, as well as anti-angiogenesis agents such as bevacizumab. Other targeted agents, including EGFR, and PARP, as well as modulators of other DNA repair enzymes, are currently tested in metastatic clinical trials and hold promise in the treatment of this aggressive disease.

Accumulating evidence suggests that $\mathrm{TN}$ tumors may be comprised of several subtypes with different molecular characteristics, natural histories, and responsiveness to treatment $[9,28-$ 31] Thus, there is a pressing need to identify the molecular basis that underlies the etiology and pathophysiology of TN tumors and to use this information to develop new therapies that specifically target tumors of this class and its putative subclasses.

\section{Acknowledgments}

The authors wish to thank the patients, physicians, nurses, data managers, and trial coordinators who participated in these studies. The authors wish to acknowledge Donna Deegan for her inspiration and Melisa Walker for editorial support. This work is supported in part by the Breast Cancer Research Foundation; National Institute of Health [Grant \# CA25224]; and The Donna Foundation. 


\section{References}

1. Dawood S, Broglio K, Kau SW, et al. Triple receptor-negative breast cancer: the effect of race on response to primary systemic treatment and survival outcomes. J Clin Oncol. 2009; 27(2):220-226. [PubMed: 19047281]

2. Irvin WJ Jr, Carey LA. What is triple-negative breast cancer? Eur J Cancer. 2008; 44(18):2799_ 2805. [PubMed: 19008097]

3. Lund MJ, Trivers KF, Porter PL, et al. Race and triple negative threats to breast cancer survival: a population-based study in Atlanta, GA. Breast Cancer Res Treat. 2009; 113(2):357-370. [PubMed: 18324472]

4. Anders C, Carey LA. Understanding and treating triple-negative breast cancer. Oncology (Williston Park). 2008; 22(11):1233-1239. discussion 1239-1240, 1243. [PubMed: 18980022]

5. Dent R, Trudeau M, Pritchard KI, et al. Triple-negative breast cancer: clinical features and patterns of recurrence. Clin Cancer Res. 2007; 13(15 Pt 1):4429-4434. [PubMed: 17671126]

6. Hines SL, Vallow LA, Tan WW, McNeil RB, Perez EA, Jain A. Clinical outcomes after a diagnosis of brain metastases in patients with estrogen- and/or human epidermal growth factor receptor 2positive versus triple-negative breast cancer. Ann Oncol. 2008; 19(9):1561-1565. [PubMed: 18534964]

7. Lin NU, Claus E, Sohl J, Razzak AR, Arnaout A, Winer EP. Sites of distant recurrence and clinical outcomes in patients with metastatic triple-negative breast cancer: high incidence of central nervous system metastases. Cancer. 2008; 113(10):2638-2645. [PubMed: 18833576]

8. Nam BH, Kim SY, Han HS, et al. Breast cancer subtypes and survival in patients with brain metastases. Breast Cancer Res. 2008; 10(1):R20. [PubMed: 18307763]

9. Rakha EA, Reis-Filho JS, Ellis IO. Basal-like breast cancer: a critical review. J Clin Oncol. 2008; 26(15):2568-2581. [PubMed: 18487574]

10. Schneider BP, Winer EP, Foulkes WD, et al. Triple-negative breast cancer: risk factors to potential targets. Clin Cancer Res. 2008; 14(24):8010-8018. [PubMed: 19088017]

11. Korsching E, Jeffrey SS, Meinerz W, Decker T, Boecker W, Buerger H. Basal carcinoma of the breast revisited: an old entity with new interpretations. J Clin Pathol. 2008; 61(5):553-560. [PubMed: 18326009]

12. Badve SS, Baehner FL, Gray RP, et al. Estrogen- and progesterone-receptor status in ECOG 2197: comparison of immunohistochemistry by local and central laboratories and quantitative reverse transcription polymerase chain reaction by central laboratory. J Clin Oncol. 2008; 26(15):24732481. [PubMed: 18487567]

13. Bryant HE, Schultz N, Thomas HD, et al. Specific killing of BRCA2-deficient tumours with inhibitors of poly(ADP-ribose) polymerase. Nature. 2005; 434(7035):913-917. [PubMed: 15829966]

14. Wolff J, Wollina U. Second malignancies in melanoma patients in Thuringia. J Eur Acad Dermatol Venereol. 2000; 14(6):479-483. [PubMed: 11444270]

15. Rakha EA, Ellis IO. Triple-negative/basal-like breast cancer: review. Pathology. 2009; 41(1):4047. [PubMed: 19089739]

16. Rakha EA, El-Sayed ME, Reis-Filho J, Ellis IO. Patho-biological aspects of basal-like breast cancer. Breast Cancer Res Treat. 2009; 113(3):411-422. [PubMed: 18327637]

17. Olopade OI, Grushko TA, Nanda R, Huo D. Advances in breast cancer: pathways to personalized medicine. Clin Cancer Res. 2008; 14(24):7988-7999. [PubMed: 19088015]

18. Parker JS, Mullins M, Cheang MC, et al. Supervised risk predictor of breast cancer based on intrinsic subtypes. J Clin Oncol. 2009; 27(8):1160-1167. [PubMed: 19204204]

19. Tan DS, Marchio C, Jones RL, et al. Triple negative breast cancer: molecular profiling and prognostic impact in adjuvant anthracycline-treated patients. Breast Cancer Res Treat. 2008; 111(1):27-44. [PubMed: 17922188]

20. Tang P, Wang J, Bourne P. Molecular classifications of breast carcinoma with similar terminology and different definitions: are they the same? Hum Pathol. 2008; 39(4):506-513. [PubMed: 18289638] 
21. Wong Z, Gerstein M, Snyder M. RNA-Seq: A revolutionary tool for transcriptomics. Nature Reviews / Genetics. 2009:1057-1063.

22. Cheang MC, Voduc D, Bajdik C, et al. Basal-like breast cancer defined by five biomarkers has superior prognostic value than triple-negative phenotype. Clin Cancer Res. 2008; 14(5):13681376. [PubMed: 18316557]

23. Elsheikh SE, Green AR, Rakha EA, et al. Caveolin 1 and Caveolin 2 are associated with breast cancer basal-like and triple-negative immunophenotype. Br J Cancer. 2008; 99(2):327-334. [PubMed: 18612310]

24. Wang S, Yang H, Tong F, et al. Response to neoadjuvant therapy and disease free survival in patients with triple-negative breast cancer. Gan To Kagaku Ryoho. 2009; 36(2):255-258. [PubMed: 19223741]

25. Eralp Y, Derin D, Ozluk Y, et al. MAPK overexpression is associated with anthracycline resistance and increased risk for recurrence in patients with triple-negative breast cancer. Ann Oncol. 2008; 19(4):669-674. [PubMed: 18006896]

26. Guler G, Huebner K, Himmetoglu C, et al. Fragile histidine triad protein, WW domain-containing oxidoreductase protein Wwox, and activator protein 2gamma expression levels correlate with basal phenotype in breast cancer. Cancer. 2009; 115(4):899-908. [PubMed: 19130459]

27. Lee CW, Simin K, Liu Q, et al. A functional Notch-survivin gene signature in basal breast cancer. Breast Cancer Res. 2008; 10(6):R97. [PubMed: 19025652]

28. Hugh J, Hanson J, Cheang MC, et al. Breast cancer subtypes and response to docetaxel in nodepositive breast cancer: use of an immunohistochemical definition in the BCIRG 001 trial. J Clin Oncol. 2009; 27(8):1168-1176. [PubMed: 19204205]

29. Rakha EA, El-Sayed ME, Green AR, Lee AH, Robertson JF, Ellis IO. Prognostic markers in triplenegative breast cancer. Cancer. 2007; 109(1):25-32. [PubMed: 17146782]

30. Reis-Filho JS, Tutt AN. Triple negative tumours: a critical review. Histopathology. 2008; 52(1): 108-118. [PubMed: 18171422]

31. Saal LH, Gruvberger-Saal SK, Persson C, et al. Recurrent gross mutations of the PTEN tumor suppressor gene in breast cancers with deficient DSB repair. Nat Genet. 2008; 40(1):102-107. [PubMed: 18066063]

32. Cortes-Funes H. The role of antiangiogenesis therapy: bevacizumab and beyond. Clin Transl Oncol. 2009; 11(6):349-355. [PubMed: 19531449]

33. Michaud LB. The epothilones: how pharmacology relates to clinical utility. Ann Pharmacother. 2009; 43(7):1294-1309. [PubMed: 19584389]

34. Andre F, Job B, Dessen P, et al. Molecular characterization of breast cancer with high-resolution oligonucleotide comparative genomic hybridization array. Clin Cancer Res. 2009; 15(2):441-451. [PubMed: 19147748]

35. Sorlie T. Introducing molecular subtyping of breast cancer into the clinic? J Clin Oncol. 2009; 27(8):1153-1154. [PubMed: 19204193]

36. Tan AR, Swain SM. Therapeutic strategies for triple-negative breast cancer. Cancer J. 2008; 14(6): 343-351. [PubMed: 19060597]

37. Liedtke C, Mazouni C, Hess KR, et al. Response to neoadjuvant therapy and long-term survival in patients with triple-negative breast cancer. J Clin Oncol. 2008; 26(8):1275-1281. [PubMed: 18250347]

38. Kelland L. The resurgence of platinum-based cancer chemotherapy. Nat Rev Cancer. 2007; 7(8): 573-584. [PubMed: 17625587]

39. Uhm JE, Park YH, Yi SY, et al. Treatment outcomes and clinicopathologic characteristics of triplenegative breast cancer patients who received platinum-containing chemotherapy. Int J Cancer. 2009; 124(6):1457-1462. [PubMed: 19065658]

40. Sirohi B, Arnedos M, Popat S, et al. Platinum-based chemotherapy in triple-negative breast cancer. Ann Oncol. 2008; 19(11):1847-1852. [PubMed: 18567607]

41. NCT00532727. [Accessed August 24] Triple Negative Breast Cancer Trial. 2009. Available at www.clinicaltrials.gov 
42. Roy V, Pockaj BA, Northfelt DW, et al. N0338 phase II trial of docetaxel and carboplatin admnistereed every two weeks as induction therapy for stage II or III breast cancer. [Abstract 563]. J Clin Oncol. 2008; 26:15S-21s.

43. NCT00782574. [Accessed August 24] Phase I/II AZD2281/Cisplatin in Neoadjuvant Treatment of Triple Negative Breast Cancer Patients. 2009. Available at www.clinicaltrials.gov

44. NCT00813956. [Accessed August 24] A Phase 2 Study of Standard Chemotherapy Plus BSI-201 (a PARP Inhibitor) in the Neoadjuvant Treatment of Triple Negative Breast Cancer. 2009. Available at www.clinicaltrials.gov

45. NCT00887575. [Accessed August 24] Neoadjuvant Sunitinib with Paclitaxel/Carboplatin in Patients with Triple-Negative Breast Cancer. 2009. Available at www.clinicaltrials.gov

46. NCT00491816. [Accessed August 24] Erlotinib Plus Chemotherapy for Treatment of Triple Negative Breast Cancer. 2009. Available at www.clinicaltrials.gov

47. Rakha EA, El-Sheikh SE, Kandil MA, El-Sayed ME, Green AR, Ellis IO. Expression of BRCA1 protein in breast cancer and its prognostic significance. Hum Pathol. 2008; 39(6):857-865. [PubMed: 18400253]

48. Turner N, Tutt A, Ashworth A. Hallmarks of 'BRCAness' in sporadic cancers. Nat Rev Cancer. 2004; 4(10):814-819. [PubMed: 15510162]

49. Hedenfalk I, Duggan D, Chen Y, et al. Gene-expression profiles in hereditary breast cancer. N Engl J Med. 2001; 344(8):539-548. [PubMed: 11207349]

50. Matros E, Wang ZC, Lodeiro G, Miron A, Iglehart JD, Richardson AL. BRCA1 promoter methylation in sporadic breast tumors: relationship to gene expression profiles. Breast Cancer Res Treat. 2005; 91(2):179-186. [PubMed: 15868446]

51. Turner NC, Reis-Filho JS, Russell AM, et al. BRCA1 dysfunction in sporadic basal-like breast cancer. Oncogene. 2007; 26(14):2126-2132. [PubMed: 17016441]

52. Kennedy RD, Quinn JE, Mullan PB, Johnston PG, Harkin DP. The role of BRCA1 in the cellular response to chemotherapy. J Natl Cancer Inst. 2004; 96(22):1659-1668. [PubMed: 15547178]

53. Carey LA, Rugo HS, Marcom PK, et al. TBCRC 001: EGFR inhibition with cetuximab added to carboplatin in metastatic triple-negative (basal-like) breast cancer. [Abstract 1009]. J Clin Oncol. 2008; 26(Suppl 15):43s.

54. O'Shaughnessy J, Weckstein DJ, Vukelja SJ, et al. Preliminary results of a randomized phase II study of weekly irinotecan/carboplatin with or without cetuximab in patients with metastatic breast cancer. Breast Cancer Res Treat. 2007; 106(Suppl 1):S32.

55. NCT00600249. [Accessed August 24] Assessment of the Efficacy of a Neoadjuvant Combination: "Chemotherapy-Targeted Therapy" in Breast Cancer. 2009. Available at www.clinicaltrials.gov

56. Rugo HS, Roche H, Thomas E, et al. Ixabepilone plus capecitabine vs. capecitabine in patients with triple negative tumors: A pool analysis of patients from two large phase III clinical studies. Cancer Res. 2008; 69(2 Suppl):3057.

57. PACS 08. http://clinicaltrials.gov/ct/show/NCT00630032.

58. Baselga J, Semiglazov V, van Dam P, et al. Phase II Randomized Study of Neoadjuvant Everolimus Plus Letrozole Compared With Placebo Plus Letrozole in Patients With Estrogen Receptor-Positive Breast Cancer. J Clin Oncol. 2009; 27(16):2630-2637. [PubMed: 19380449]

59. Campone, M.; Tan, WW.; Perez, EA. [Accessed April] Randomized, open label, multicenter phase III evaluating the benefit of a sequential regimen associating FEC 100 and Ixabepilone in adjuvant treatment of non metastatic, poor prognosis breast cancer defined as triple-negative tumor [HER2 negative - ER negative - PR netagive] or [HER2 negative and PR negative] tumor; in node positive or node negative patients. 2009. Available at http://www.clinicaltrial.gov/ct2/show/ NCT00630032?term=NCT00630032\&rank=1.

60. Titan. http://clinicaltrials.gov/ct/show/NCT00789581.

61. Yardley, DA. [Accessed April] Phase III study of doxorubicin/cyclophosphamide (AC) followed by Ixabepilone vs. AC followed by paclitaxel in patients with triple-negative early-stage breast cancer. 2009. Available at http://www.clinicaltrial.gov/ct2/show/NCT00789581? term $=$ NCT00789581\&rank=1.

62. GEICAM 2006-03. http://clinicaltrials.gov/ct/show/NCT00432172. 
63. Alba, E.; Albanell, J.; Adrover, E., et al. [Accessed April] A randomized multicenter phase II trial to evaluate the effectiveness of selective neoadjuvant treatment according to immunohistochemical subtype for HER2 negative breast cancer patients. 2009. Available at http://www.clinicaltrail.gov/ ct2/show/NCT00432172?term=NCT00432172\&rank=1.

64. CALGB 40603. http://clinicaltrials.gov/ct/show/NCT00861705.

65. Sikov, WM. [Accessed April] Randomized phase II $2 \times 2$ factorial trial of the addition of carboplatin +/- bevacizumab to neoadjuvant weekly paclitaxel followed by dose- dense AC in hormone receptor-poor/HER2-negative resectable breast cancer. 2009. Available at http:// www.clinicaltrial.gov/ct2/show/NCT00861705?term=NCT00861705\&rank=1.

66. BEATRICE. http://clinicaltrials.gov/ct/show/NCT0000528567.

67. Korkaya H, Wicha MS. HER-2, notch, and breast cancer stem cells: targeting an axis of evil. Clin Cancer Res. 2009; 15(6):1845-1847. [PubMed: 19276254]

68. Raouf A, Zhao Y, To K, et al. Transcriptome analysis of the normal human mammary cell commitment and differentiation process. Cell Stem Cell. 2008; 3(1):109-118. [PubMed: 18593563]

69. Perez EA, Suman VJ, Davidson NE, et al. Cardiac safety analysis of doxorubicin and cyclophosphamide followed by paclitaxel with or without trastuzumab in the North Central Cancer Treatment Group N9831 adjuvant breast cancer trial. J Clin Oncol. 2008; 26(8):1231-1238. [PubMed: 18250349]

70. Ratnam K, Low JA. Current development of clinical inhibitors of poly(ADP-ribose) polymerase in oncology. Clin Cancer Res. 2007; 13(5):1383-1388. [PubMed: 17332279]

71. Voduc D, Nielsen TO. Basal and triple-negative breast cancers: impact on clinical decision-making and novel therapeutic options. Clin Breast Cancer. 2008; 8(Suppl 4S):171-178. 


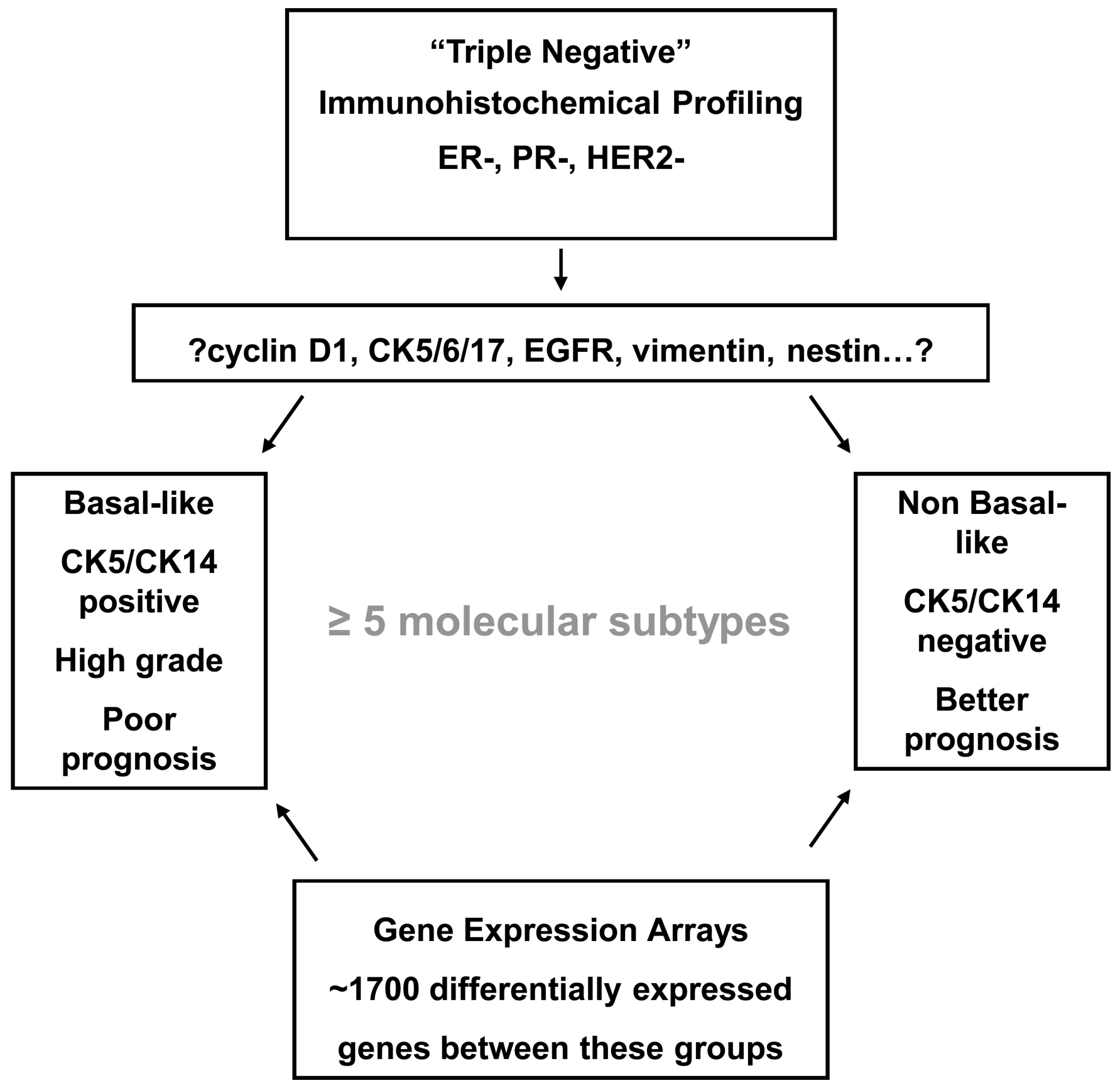

Figure 1. "Triple Negative" Disease in the Context of "Basal-like" Breast Cancer Classification of so called triple negative breast cancers is beginning to be modified by inclusion of immunohistochemistry for various markers. Triple negative breast cancers exhibit a range of molecular and clinical properties that suggest that they are comprised of several subtypes. There is a tendency to equate basal-like tumors with triple negative due to ER/PR negative status and no amplification and/or overexpression of HER2, yet as many as $10-20 \%$ of triple negative tumors exhibit a non-basal genomic profile and there appear to be at least 5 molecular subtypes. Evaluating the molecular characteristics, between the different subtypes may be essential to understanding the natural histories and their responsiveness to treatment. 


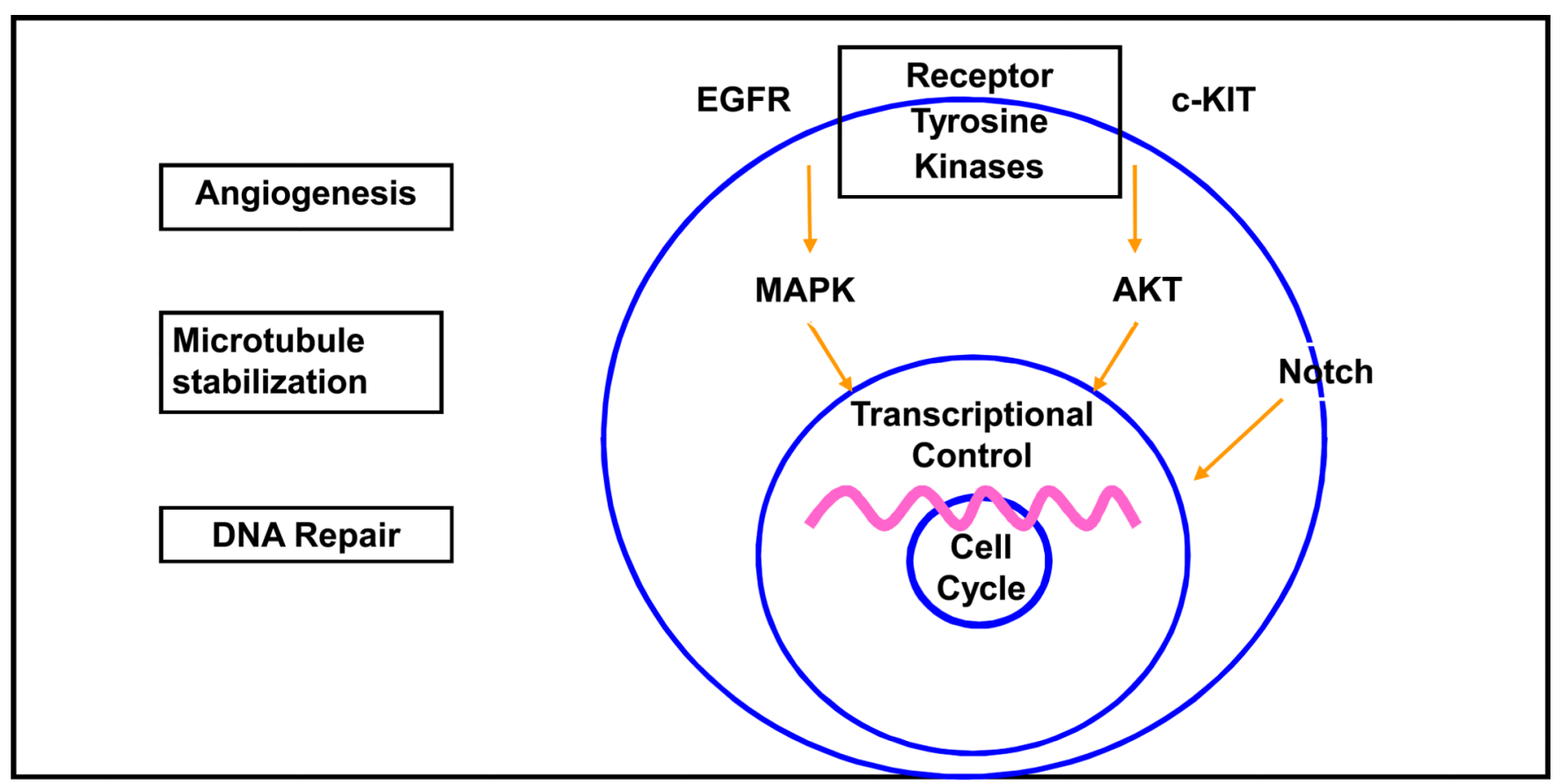

Figure 2. Potential Therapeutic Targets for Triple Negative Breast Cancer [27, 36, 70, 71] The majority of therapeutic targets currently under investigation fall within three broad categories: anti-angiogenesis (bevacizumab and other anti-VEGF compounds), stabilization of microtubules (MSAs such as ixabepilone) and deoxyribonucleic acid (DNA) repair (including platinums and PARP inhibitors, such as olaparib and BSI-120). 


\section{Table 1}

Triple Negative Breast Cancer: Why Consider Optimizing Adjuvant Treatment [4-10]?

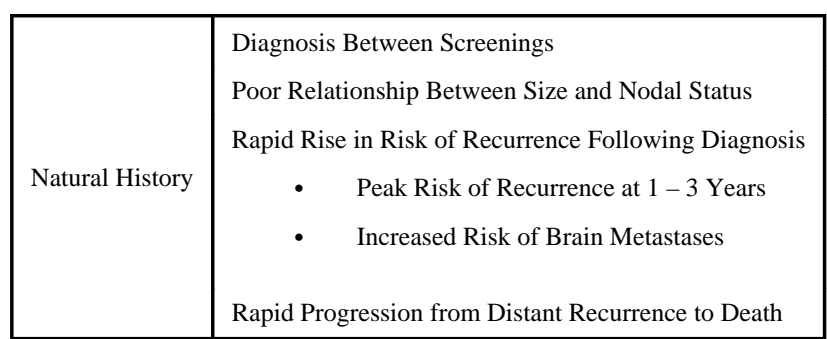

